\title{
Eletroestimulação no tratamento fisioterapêutico da bexiga neurogênica: uma revisão integrativa
}

\author{
Electrostimulation in the physiotherapeutic treatment of neurogenic bladder: an integrative review \\ Electroestimulación en el tratamiento fisioterapéutico de la vejiga neurogénica: una revisión
}

integradora

Recebido: 04/10/2021 | Revisado: 10/10/2021 | Aceito: 12/10/2021 | Publicado: 14/10/2021

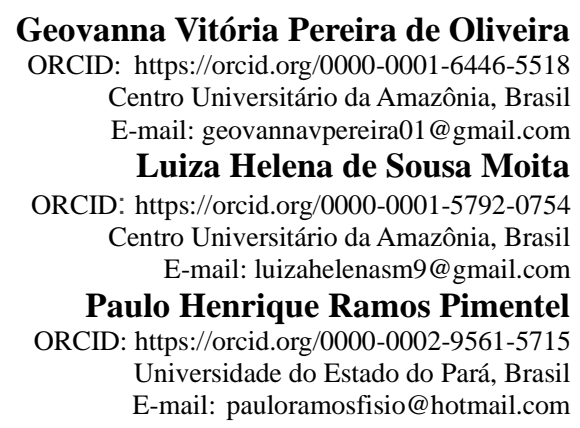

\begin{abstract}
Resumo
Disfunção do trato urinário inferior (DNTUI) ou também conhecida como Bexiga Neurogênica (BN), refere-se aos distúrbios miccionais ocasionados por afecções neurológicas no Sistema Nervoso Central ou Periférico, onde estes provocam alterações dos padrões miccionais dos pacientes, principalmente nas inervações vesico-esfincterianas, e nas fases de enchimento ou esvaziamento vesical. Dentre as intervenções fisioterapêuticas, a eletroestimulação apresenta-se como forma de tratamento viável, com bons resultados, fácil aplicação, baixo custo e inexistência de efeitos colaterais.Os objetivos desta revisão é investigar a eficácia da eletroestimulação em pacientes com bexiga neurogênica em variados distúrbios neurológicos. Foram utilizadas as bases de dados eletrônicas PubMed e SciELO. Foram selecionados 10 estudos para a realização dessa revisão. Os resultados demonstraram que a eletroestimulação no nervo tibial posterior foi utilizado em $70 \%$ dos estudos, aparecendo de forma majoritária enquanto o uso da eletroestimulação no nervo parassacral, foi citado em $30 \%$ dos artigos. A frequência mais utilizada foi de $20 \mathrm{~Hz}$ e a largura de pulso variou entre 200 e $500 \mu$ s. Os resultados mostraram diminuição dos sintomas da bexiga neurogênica, regressão de perdas urinárias e impactaram no tempo de enchimento vesical. Portanto, foi demonstrado que os nervos tibial posterior e parassacral, por serem de fácil acesso, ajudam na execução do tratamento da bexiga neurogênica, tornando-o seguro e indolor.
\end{abstract}

Palavras-chave: Especialidade de fisioterapia; Estimulação elétrica nervosa transcutânea; Bexiga urinária neurogênica.

\begin{abstract}
Lower Urinary Tract Dysfunction (DNTUI) or also known as Neurogenic Bladder (BN), refers to voiding disorders caused by neurological disorders in the Central or Peripheral Nervous System, where they cause changes in patients' voiding patterns, especially in the bladder innervations. sphincter, and in the filling or emptying phases. Among the physical therapy interventions, electrical stimulation is a viable form of treatment, with good results, easy application, low cost and no side effects. The objectives of this review is to investigate the effectiveness of electrical stimulation in patients with neurogenic bladder in various neurological disorders . The electronic databases PubMed and SciELO were used. 10 studies were selected to carry out this review. The results showed that electrical stimulation in the posterior tibial nerve was used in $70 \%$ of the studies, appearing in the majority, while the use of electrical stimulation in the parasacral nerve was mentioned in $30 \%$ of the articles. The most used frequency was $20 \mathrm{~Hz}$ and the pulse width ranged between 200 and $500 \mu$ s. The results showed a decrease in neurogenic bladder symptoms, regression of urinary losses and had a impact on bladder filling time. Therefore, it has been shown that the posterior tibial and parasacral nerves, as they are easily accessible, help in carrying out the treatment of the neurogenic bladder, making it safe and painless.
\end{abstract}

Keywords: Physiotherapy specialty; Transcutaneous electrical nerve stimulation; Neurogenic urinary bladder. 


\section{Resumen}

La disfunción del tracto urinario inferior (DNTUI) o también conocida como vejiga neurogénica (BN), se refiere a los trastornos miccionales causados por trastornos neurológicos en el sistema nervioso central o periférico, donde provocan cambios en los patrones de micción de los pacientes, especialmente en las inervaciones de la vejiga, y en las fases de llenado o vaciado. Entre las intervenciones de fisioterapia, la electroestimulación es una forma viable de tratamiento, con buenos resultados, fácil aplicación, bajo costo y sin efectos secundarios. El objetivo de esta revisión es investigar la efectividad de la electroestimulación en pacientes con vejiga neurogénica en diversas enfermedades neurológicas. trastornos. Se utilizaron las bases de datos electrónicas PubMed y SciELO. Se seleccionaron diez estudios para realizar esta revisión. Los resultados mostraron que la estimulación eléctrica en el nervio tibial posterior se utilizó en el $70 \%$ de los estudios, apareciendo en la mayoría, mientras que el uso de estimulación eléctrica en el nervio parasacro se mencionó en el 30\% de los artículos. La frecuencia más utilizada fue de $20 \mathrm{~Hz}$ y el ancho de pulso osciló entre 200 y $500 \mu$ s. Los resultados mostraron una disminución en los síntomas de la vejiga neurogénica, regresión de las pérdidas urinarias y tuvieron un impacto en el tiempo de llenado de la vejiga. Por tanto, se ha demostrado que los nervios tibial posterior y parasacro, al ser de fácil acceso, ayudan en la realización del tratamiento de la vejiga neurogénica, haciéndola segura e indolora.

Palabras clave: Especialidad de fisioterapia; Estimulación nerviosa eléctrica transcutánea; Vejiga urinaria neurogênica.

\section{Introdução}

As complicações do trato urinário afetam diretamente a qualidade de vida dos pacientes neurológicos (Hearn et al., 2018). Disfunção do trato urinário inferior (DNTUI) ou também conhecida como Bexiga Neurogênica (BN), refere-se aos distúrbios miccionais ocasionados por afecções neurológicas no Sistema Nervoso Central ou Periférico, onde estes provocam alterações dos padrões miccionais dos pacientes, principalmente nas inervações vesico-esfincterianas, e nas fases de enchimento ou esvaziamento vesical (Dias, 2016). Pacientes com distúrbios do Sistema Nervoso, na maioria das vezes, apresentam bexiga neurogênica (Schops et al., 2015). Dentre os distúrbios, destacam-se a lesão medular, esclerose múltipla, doença de Parkinson, paralisia cerebral e acidente vascular encefálico (Jahromi et al., 2014). Dependendo do déficit neurológico, a BN pode ser hipoativa; sem capacidade de contração voluntária, ou hiperativa; com perda involuntária de urina (Liao, 2015). A atividade correta de micção depende da sinergia entre a bexiga e o esfíncter urinário. Nesse processo, a bexiga permanece relaxada e com pressão baixa durante a fase de enchimento. Já na fase de esvaziamento ocorre uma contração da bexiga, pois sua capacidade é atingida (Monteiro et al., 2017).

A anamnese, exame físico, exames de imagem, estudos urológicos e urodinâmicos, estão entre as principais formas de diagnóstico da bexiga neurogênica, sendo o último o mais importante. Para o melhor diagnóstico destas disfunções, o uso de questionários de sintomas estruturados tornou-se uma ferramenta indispensável e um instrumento valioso no manejo do tratamento de pacientes com sintomas do trato urinário inferior (Tsang et al., 2016). Já entre complicações da BN, tem-se infecções do trato urinário, refluxo uretral e formação de cálculos renais (Lue e Tanagho, 2014).

A BN torna-se um grande obstáculo para a saúde pública devido ao grande acervo de pessoas afetadas, gerando grandes gastos nos sistemas de saúde, além de impactar negativamente na qualidade de vida dos pacientes (Schneider et al., 2015). Tendo em vista esses impactos, faz-se necessário um acompanhamento multidisciplinar, afim de traçar um plano terapêutico mais amplo e direcionado ao processo de condição de saúde, para atender as necessidades individuais de cada paciente (Leal, 2018).

Dentre as formas de tratamento para a BN, destacam-se os medicamentosos, invasivos e fisioterapêuticos. Em relação aos medicamentosos, existem drogas que podem causar efeitos colaterais e até mesmo ineficácia em relação ao tratamento. Já os procedimentos invasivos, como os cirúrgicos, podem acarretar complicações para os pacientes (Starkman et al., 2010). No entanto, o tratamento fisioterapêutico mostrou-se seguro e eficaz, preservando o trato urinário superior e impedindo futuras infecções urinárias posteriores ao tratamento (Liao, 2015). Dentre as intervenções fisioterapêuticas, a eletroestimulação apresenta-se como forma de tratamento viável, com bons resultados, fácil aplicação, baixo custo e 
inexistência de efeitos colaterais (Kabay et al., 2016; Perissinotto et al., 2015).

Estudos mostram que há um grande acervo de aplicabilidade dentro da eletroestimulação, desde o tipo de corrente, até a colocação de eletrodos (Radziszewski, 2013). A eletroestimulação transcutânea (TENS), é realizada através dos eletrodos de superfície, sendo aplicada em diversas regiões, como: genital, hipogástrica, sacral e intravesical (Carr, 2010). O Sistema Nervoso Central (SNC) pode ter suas estruturas ativadas através dessa intervenção, na qual é utilizada uma corrente elétrica que pode modular a inervação da bexiga, melhorando, assim, os sintomas da BN. Os plexos nervosos pélvico e sacral são os responsáveis por inervar o trato urinário inferior, e segundo a literatura, esse modelo de estimulação elétrica demonstra bons resultados no tratamento desses plexos (Guys et al., 2004)

Dessa forma, o objetivo dessa revisão é investigar a eficácia clínica da eletroestimulação em pacientes com BN em variados distúrbios neurológicos.

\section{Metodologia}

Uma revisão de literatura do tipo integrativa compõe um método específico de pesquisa que busca analisar e compreender de forma mais abrangente fatos sobre um determinado fenômeno (Botelho et al., 2011). A revisão integrativa oportuniza a crítica, a comparação e a avaliação de diversas evidências científicas sobre um tema em questão, gerando resultados que demonstram o grau de conhecimento acerca do tema estudado (Mendes et al., 2008).

Trata-se de uma revisão integrativa de literatura, com busca nas bases de dados eletrônicas PubMed e SciELO. As buscas foram feitas no idioma inglês, com os seguintes descritores: "physiotherapy specialty", "transcutaneous electrical nerve stimulation" and "neurogenic urinary bladder". Esses descritores foram consultados por meio do DECS (Descritores de assuntos em ciências da saúde da BIREME). Nessa revisão foram incluídos estudos originais indexados nos últimos oito anos (2013-2020) na língua inglesa e portuguesa, disponíveis em formato de texto completo e que abordassem a eletroestimulação em pacientes neurológicos com sintomas de bexiga neurogênica. Foram excluídos artigos com pesquisas em animais, estudos de revisões, estudos de caso, estudos que não tiveram coerência com o tema, artigos elaborados em outros idiomas que não o elegido e com datas de publicação inferiores a oito anos.

Primeiramente foi indagada a seguinte pergunta: "A eletroterapia é eficaz no tratamento da bexiga neurogênica?". Posteriormente, para a elaboração dessa pesquisa, foi delimitado o assunto a ser abordado, em seguida, foi realizado um levantamento de estudos indexados nas bases de dados eletrônicas selecionadas. Foram excluídos artigos repetidos, artigos que não tiveram coerência com o objetivo dessa revisão após a leitura dos títulos e resumos, e artigos que não possuíam os critérios de elegibilidade delimitados para essa pesquisa.

\section{Resultados e Discussão}

Foram encontrados 891 artigos nas bases de dados eletrônicas selecionadas. Depois da avaliação, leitura dos resumos e títulos, aplicação dos critérios de inclusão e exclusão e leitura completa dos artigos, foram selecionados 10 estudos para a realização dessa revisão. As características de cada estudo estão dispostas no Quadro 1. 
Quadro 1- artigos selecionados para a presente revisão.

\begin{tabular}{|c|c|c|c|c|}
\hline Autores & Objetivo & Amostra & Intervenção & Conclusão \\
\hline Araújo, 2020. & $\begin{array}{l}\text { Verificar a eficácia da } \\
\text { EENT (estimulação } \\
\text { elétrica no nervo tibial) } \\
\text { em pacientes com BN } \\
\text { (Bexiga neurogênica) } \\
\text { pós AVE Acidente } \\
\text { vascular encefálico). }\end{array}$ & $\begin{array}{l}11 \text { mulheres de } 40 \text { a } 70 \\
\text { anos diagnosticadas } \\
\text { com AVE. }\end{array}$ & $\begin{array}{l}\text { Foi inserido o TENS } \\
\text { (Transcutaneous Electrical } \\
\text { Nerve Stimulation) no } \\
\text { nervo tibial duas vezes na } \\
\text { semana, durante } 12 \\
\text { semanas com um período } \\
\text { de } 30 \text { minutos, frequência } \\
\text { de } 10 \mathrm{~Hz} \text { e largura de pulso } \\
\text { de } 200 \mu \mathrm{s} \text {. }\end{array}$ & $\begin{array}{l}\text { A eletroestimulação mostrou-se } \\
\text { eficaz, reduzindo as perdas } \\
\text { urinárias e melhorando a } \\
\text { qualidade de vida das pacientes. }\end{array}$ \\
\hline $\begin{array}{l}\text { Dombek, } \\
2019\end{array}$ & $\begin{array}{l}\text { Avaliar o efeito do } \\
\text { TENS na região } \\
\text { parassacral em crianças } \\
\text { com BN portadoras de } \\
\text { mielomeningocele. }\end{array}$ & $\begin{array}{l}26 \text { crianças entre } 5 \text { a } 15 \\
\text { anos com baixa } \\
\text { complacência vesical. }\end{array}$ & $\begin{array}{l}\text { Foi utilizado o Tens na } \\
\text { região parassacral com } \\
\text { freqência de } 10 \mathrm{~Hz} \text { e } \\
\text { largura de pulso de } 500 \mu \mathrm{s} \text {. }\end{array}$ & $\begin{array}{l}\text { O TENS parassacral reduziu a } \\
\text { pressão máxima da bexiga nos } \\
\text { estudos urodinâmicos, e obteve } \\
\text { um resultado melhor em crianças } \\
\text { menores de } 12 \text { anos. }\end{array}$ \\
\hline $\begin{array}{l}\text { Stampas et } \\
\text { al., } 2018 \text {. }\end{array}$ & $\begin{array}{l}\text { Investigar a TTNS } \\
\text { (Transcutaneous tibial } \\
\text { nerve stimulation) em } \\
\text { pacientes com BN } \\
\text { portadores de lesão na } \\
\text { medula espinhal. }\end{array}$ & $\begin{array}{l}19 \text { pacientes } \\
\text { hospitalizados com } \\
\text { idade entre } 18-65 \text { anos. }\end{array}$ & $\begin{array}{l}\text { Os pacientes receberam a } \\
\text { TTNS durante } 30 \text { minutos } \\
\text { durante } 2 \text { semanas. Os } \\
\text { eletrodos } \\
\text { posicionados na perna } \\
\text { direita com o eletrodo } \\
\text { negativo atrás do maléolo } \\
\text { medial e o eletrodo positivo } \\
10 \mathrm{~cm} \text { superior ao negativo, } \\
\text { com frequência de } 10 \mathrm{~Hz} \text { e } \\
\text { largura de pulso de } 200 \mu \mathrm{s} \text {. }\end{array}$ & $\begin{array}{l}\text { O TTNS mostrou eficaz em } \\
\text { pacientes com lesão medular, } \\
\text { aumentando o tempo de } \\
\text { enchimento vesical. }\end{array}$ \\
\hline $\begin{array}{l}\text { Cabaz et al., } \\
2017 .\end{array}$ & $\begin{array}{l}\text { Determinar a eficácia da } \\
\text { PTNS (Percutaneous } \\
\text { Tibial Nerve } \\
\text { Stimulation) } \quad \text { em } \\
\text { pacientes com esclerose } \\
\text { múltipla. }\end{array}$ & $\begin{array}{l}21 \text { pacientes maiores de } \\
18 \text { anos com } \\
\text { diagnóstico } \\
\text { esclerose múltipla }\end{array}$ & $\begin{array}{l}\text { Foi realizada a terapia } \\
\text { durante } 1 \text { ano com um } \\
\text { protocolo de redução } \\
\text { gradual de } 6,9 \text { e } 12 \text { meses, } \\
\text { unilateralmente com uma } \\
\text { frequência de } 20 \mathrm{~Hz} \text { e } \\
\text { largura de pulso de } 200 \mu \mathrm{s} \text {. } \\
5 \mathrm{~cm} \text { acima do maléolo } \\
\text { medial e posterior à borda } \\
\text { da tíbia. Foi realizado } \\
\text { também um diário } \\
\text { miccional de } 3 \text { dias } \\
\text { inicialmente e aos } 3,6,9 \text { e } \\
12 \text { meses. }\end{array}$ & $\begin{array}{l}\text { Os pacientes obtiveram uma } \\
\text { melhora significativa da } \\
\text { frequência, noctúria, episódios de } \\
\text { urgência e do volume esvaziado }\end{array}$ \\
\hline $\begin{array}{l}\text { Araújo, } \\
2017 .\end{array}$ & $\begin{array}{l}\text { Comparar os efeitos do } \\
\text { ENTP (estimulação no } \\
\text { nervo tibial posterior) } \\
\text { em pacientes com } \\
\text { doença de Parkinson. }\end{array}$ & $\begin{array}{l}\text { Mulheres com doença } \\
\text { de parkinson. }\end{array}$ & $\begin{array}{l}\text { Foi utilizado a ENTP } \\
\text { domiciliar, além disso } \\
\text { foram aplicados } \\
\text { questionários de avaliação } \\
\text { da incontinência urinária e } \\
\text { qualidade de vida (OAB- } \\
\text { V8 e KHQ) e de um diário } \\
\text { miccional de } 24 \text { horas, }\end{array}$ & $\begin{array}{l}\text { O tratamento mostrou-se eficaz, } \\
\text { diminuindo episódios de } \\
\text { noctúria, número de episódios de } \\
\text { urgência miccional e urge- } \\
\text { incontinência e número de uso de } \\
\text { proteções para incontinência. }\end{array}$ \\
\hline
\end{tabular}




\begin{tabular}{|c|c|c|c|c|}
\hline $\begin{array}{l}\text { Kabay et al., } \\
2016 .\end{array}$ & $\begin{array}{l}\text { Investigar a eficácia da } \\
\text { PTNS em pacientes com } \\
\text { bexiga neurogênica } \\
\text { portadores da doença de } \\
\text { Parkinson. }\end{array}$ & $\begin{array}{l}\text { Foram incluidos } 47 \\
\text { pacientes com doença } \\
\text { de parkinson com } \\
\text { sintomas de BN. }\end{array}$ & $\begin{array}{l}\text { Foi realizada durante } 12 \\
\text { semanas a estimulação no } \\
\text { nervo tibial posterior } \\
\text { unilateralmente, } \quad \text { com } \\
\text { largura de pulso de } 200 \mu \mathrm{s} \mathrm{e} \\
\text { Frequência de } 20 \mathrm{~Hz} \text { A A } \\
\text { intensidade foi ditada pelo } \\
\text { paciente. }\end{array}$ & $\begin{array}{l}\text { Os resultados mostraram que a } \\
\text { PTNS melhora os sintomas de } \\
\text { bexiga neurogênica e os } \\
\text { parâmetros urodinâmicos }\end{array}$ \\
\hline $\begin{array}{l}\text { Chen et al., } \\
2015\end{array}$ & $\begin{array}{l}\text { Eficácia da PTNS em } \\
\text { pacientes com lesão } \\
\text { medular. }\end{array}$ & $\begin{array}{l}100 \text { pacientes maiores } \\
\text { de } 18 \text { anos com lesão } \\
\text { medular com } \\
\text { diagnóstico de BN. }\end{array}$ & $\begin{array}{l}\text { Foram divididos em grupo } \\
\text { A e B com } 50 \text { pacientes em } \\
\text { cada. No grupo A, os } \\
\text { pacientes receberam PTNS } \\
\text { no nervo tibial, } 2 \text { vezes na } \\
\text { semana por } 4 \text { semanas } \\
\text { durante } 30 \text { minutos, com } \\
\text { frequência de } 1 \mathrm{~Hz} \text { e } \\
\text { largura de pulso } 10 \mu \mathrm{s} \text {. No } \\
\text { grupo } \mathrm{B} \text {, os pacientes foram } \\
\text { submetidos à frequência de } \\
20 \mathrm{~Hz} \text { e largura de pulso de } \\
200 \mu \text { s durante } 4 \text { semanas }\end{array}$ & $\begin{array}{l}\text { A PTNS melhorou a qualidade de } \\
\text { vida dos pacientes estudados. }\end{array}$ \\
\hline $\begin{array}{l}\text { Ojha et al,. } \\
2015 .\end{array}$ & $\begin{array}{l}\text { Verificar a eficácia da } \\
\text { PTN (nervo tibial } \\
\text { posterior) e DPN (nervo } \\
\text { peniano dorsal) em } \\
\text { pacientes com lesão } \\
\text { medular diagnosticadas } \\
\text { com BN. }\end{array}$ & $\begin{array}{l}15 \text { pacientes foram } \\
\text { estudados. } 10 \text { pacientes } \\
\text { com onda } \mathrm{F} \text { e } 5 \\
\text { pacientes com ausência } \\
\text { de onda } \mathrm{F} \text {, no qual } 9 \\
\text { eram homens e } 1 \\
\text { mulher, } \\
6 \text { tinham lesão medular } \\
\text { completa e } 4 \\
\text { incompleta. }\end{array}$ & $\begin{array}{l}\text { Foi aplicada uma } \\
\text { estimulação de } 20 \mathrm{~Hz}, 10- \\
40 \mathrm{~mA} \text { por } 20 \text { minutos por } \\
14 \text { dias consecutivos. }\end{array}$ & $\begin{array}{l}\text { Os gráficos de micção mostraram } \\
\text { melhora signiticativa em ambos } \\
\text { os grupos. }\end{array}$ \\
\hline $\begin{array}{l}\text { Knupfer et } \\
\text { al,. } 2014 \text {. }\end{array}$ & $\begin{array}{lr}\text { Eficácia } & \text { da } \\
\text { neuromodulação } & \text { sacral } \\
\text { em pacientes com } & \text { BN. }\end{array}$ & $\begin{array}{l}30 \text { pacientes maiores de } \\
18 \text { anos com variados } \\
\text { distúrbios neurológicos. }\end{array}$ & $\begin{array}{lr}\text { Foram } & \text { introduzidos } \\
\text { eletrodos bilateralmente em } \\
\text { s3 e s4, durante } 3-6 \\
\text { semanas. }\end{array}$ & $\begin{array}{l}\text { A neromodulação sacral } \\
\text { apresentou ótimos resultados } \\
\text { como forma de tratamento em } \\
\text { pacientes com BN. }\end{array}$ \\
\hline $\begin{array}{l}\text { Nepomucen } \\
\text { o, } 2013 \text {. }\end{array}$ & $\begin{array}{l}\text { Comparar a eficácia do } \\
\text { TENS parassacral em } \\
\text { relação a oxibutinina em } \\
\text { crianças portadoras de } \\
\text { bexiga neurogênica. }\end{array}$ & $\begin{array}{l}26 \text { crianças com } \mathrm{BN} \\
\text { com idade média de } 6,4 \\
\text { a } 4,2 \text { anos }\end{array}$ & $\begin{array}{l}18 \text { crianças receberam o } \\
\text { tratamento com oxibutinina } \\
\text { oral por } 10 \text { semanas e } 8 \\
\text { crianças foram submetidas } \\
\text { ao tratamento do TENS } \\
\text { parassacral durante } 30 \\
\text { sessões. }\end{array}$ & $\begin{array}{l}\text { O TENS parassacral melhorou a } \\
\text { continência em } 87,5 \% \text { das } \\
\text { crianças, enquanto que a } \\
\text { oxibutinina melhorou } 66,67 \% \text { a } \\
\text { continência. Ambos melhoraram } \\
\text { a complacência vesical e } \\
\text { diminuiram o número de fraldas, } \\
\text { porém, a maior parte dos } \\
\text { pacientes que utilizaram a TENS } \\
\text { apresentaram sensibilidade ao } \\
\text { enchimento vesical. }\end{array}$ \\
\hline
\end{tabular}

Fonte: Oliveira, Moita e Pimentel (2021).

Em 1791 foram descobertas as primeiras percepções do uso da eletroestimulação como forma de tratamento. Luigi Galvani foi percursor deste marco, identificando que correntes elétricas poderiam gerar contrações musculares por meio de um determinado potencial de ação. Com isso, em 1833, Duchene de Boulogne usou a descoberta de Galvani para aplicar o 
método em portadores de paralisia muscular, desvendando então, a possibilidade de estimular músculos com correntes elétricas sem a necessidade de perfurar o tecido epitelial e criou eletrodos para realizar a estimulação percutânea. Assim, Duchene de Boulogne foi considerado pai da eletroterapia (Mcdonough e Kitchen, 2003).

A eletroestimulação na bexiga ainda não tem seus mecanismos fisiológicos completamente esclarecidos, porém no que tange o tratamento de disfunções miccionais, acredita-se que aconteça uma reorganização das ações dos impulsos excitatórios e inibitórios, revertendo ou recuperando a função deste órgão. Com isso, o princípio da eletroestimulação denota que as estruturas neurais do sistema nervoso central se ativam ao receberem estímulos de uma corrente elétrica, gerando modulações nos nervos da bexiga (Rocha, 2009; Maternik et al., 2015).

No que se refere ao local de aplicação da eletroestimulação o nervo tibial posterior foi utilizado em 07 estudos, somando $70 \%$ dos achados, aparecendo de forma majoritária. O nervo tibial posterior é descrito na literatura como um nervo de função mista, abrangendo as fibras de L4-S3 que por sua vez possuem origem nas mesmas raízes nervosas da bexiga e de todo assoalho pélvico, sendo assim a EENT é capaz de gerar reflexos inibitórios nos neurônios espino-talâmicos causando inibição das contrações involuntárias do musculo detrusor, também diminuindo perdas urinárias e melhorando os sintomas da bexiga neurogênica (Zecca et al., 2016). Em seguida, os estudos demostraram o uso da eletroestimulação no nervo parassacral, este sendo citado em $30 \%$ dos artigos estudados. Este nervo funciona como modulador das vias neurais aferentes e eferentes no arcabouço do assoalho pélvico, principalmente bexiga e uretra, com isso, acredita-se que o uso da eletroestimulação nas raízes sacrais, especificamente em S3, cause inibição das atividades do músculo detrusor, levando a uma melhora significativa da capacidade de armazenamento da bexiga e diminuindo escapes urinários (Leng e Chancellor, 2005).

Repercutindo os achados na melhora do quadro clínico dos pacientes estudados, os artigos selecionados mostraram que o uso da eletroestimulação foi benéfica em todos os casos. Os artigos evidenciaram a prevalência das doenças neurológicas em pacientes com diagnóstico de bexiga neurogênica, sendo lesão medular somando 30\% dos achados, seguidos de pacientes com doença de Parkinson; somando 20\% dos estudos, pesquisas com crianças 20\%, Acidente Vascular Encefálico 10\%, Esclerose Múltipla 10\% e um estudo com distúrbios neurológicos variados somando 10\%. No estudo de Dombek, 2019 e Nepomuceno et al., 2013 foi verificado o uso da eletroestimulação do tipo TENS no nervo parassacral em crianças com bexiga neurogênica. A intervenção mostrou-se eficaz em ambos os estudos, demostrando que as crianças do grupo amostral tiveram melhoras nos sintomas urinários, diminuindo a pressão urinária e o tempo de enchimento e aumentando a sensibilidade vesical.

Stampas et al., 2018 verificou o uso da eletroestimulação no nervo tibial posterior em pacientes com bexiga neurogênica pós lesão medular e constatou um aumentou no tempo de enchimento vesical em seu grupo de estudo. Corroborando com estes fatos, os estudos de Chen et al, 2015 e Ojha et al., 2015 também pesquisaram sobre os efeitos da eletroestimulação no nervo tibial posterior em pacientes com sintomas urinários após lesão medular, ambos constataram melhora nos sintomas e na qualidade de vida dos pacientes estudados. Os autores De Araujo, 2017 e Kabay et al., 2016 evidenciaram em suas pesquisas que pacientes com Parkinson tiveram resultados positivos utilizando a eletroestimulação no nervo tibial posterior, gerando resultados positivos quanto as perdas de urina de emergência e urge-incontinencia, além de melhora na qualidade de vida do grupo amostral. Nos demais artigos a eletroestimulação também foi vantajosa aos pacientes, melhorando principalmente as perdas urinárias, diminuindo a noctúria e frequência das micções, além de beneficiar sua qualidade de vida, uma vez que mal humor, baixa autoestima e distúrbios do sono são algumas consequências associadas ao diagnóstico de bexiga neurogênica, impactando de forma negativa na vida dos pacientes e afetando seu bem estar físico, psicológico e social (Zecca et al., 2016). 
No que se relaciona aos parâmetros utilizados houve variação entre os autores, sendo que a frequência de $20 \mathrm{~Hz}$ foi utilizada em $40 \%$ dos estudos, enquanto que os demais utilizaram frequência fixa de $10 \mathrm{~Hz}$ somando $30 \%$, os demais autores não informaram as frequências utilizadas. Quanto a largura de pulso, esta variou entre a mínima de $200 \mu$ s, utilizadas de forma majoritária (50\%) pelos autores, e a máxima de $500 \mu$ s usada apenas por 01 autor, os demais trabalhos não informaram a largura de pulso de suas intervenções. As correntes elétricas podem impactar diretamente o sistema nervoso central, deixando-o propício e facilitando o fenômeno da neuroplasticidade e as atividades aferentes e eferentes do trato urinário inferior, com isso os parâmetros elétricos precisam ser utilizados de maneira correta, alguns estudos constataram que a baixa frequência, 5 a 20Hz, inibem contrações involuntárias da bexiga. Além disso, autores verificaram que a melhor frequência no tratamento da bexiga hiperativa é acima de $10 \mathrm{~Hz}$, podendo ser usado também no tratamento da bexiga neurogênica (Stepherson e Shelly, 2010; Nijman, 2016; Barroso et al., 2011).

\section{Considerações Finais}

Portanto, verificou-se que o uso da eletroestimulação é uma importante ferramenta no tratamento da bexiga neurogênica. Seus parâmetros, locais e tempos de aplicação melhoraram significativamente os sintomas dos pacientes estudados. Os nervos tibial posterior e parassacral, por serem de fácil acesso, ajudam na execução do tratamento tornando-o seguro e indolor.

Contudo, faz-se necessário a elaboração de mais estudos que fomentem as discussões acerca do tema, reforçando a necessidade de um bom tratamento para portadores de bexiga neurogênica. As futuras pesquisas devem abordar sobre o tema em questão, trazendo à tona os benefícios de um tratamento precoce, desvinculando episódios de perda urinaria como aspectos normais do cotidiano e alertando sobre as consequências que a bexiga neurogênica, se não tratada, pode trazer aos indivíduos. Consequências estas não somente físicas, mas psicológicas e sociais, que impactam diretamente nas relações interpessoais dos portadores desta doença.

\section{Agradecimentos}

Agradecemos a Deus por nos sustentar, por nos dar resiliência e persistência diante das dificuldades. Agradecemos as nossas famílias por serem nossas bases de amor e confiança.

\section{Referências}

Araujo, R.C. (2020). Efeitos da técnica de eletroestimulação do nervo tibial em mulheres com bexiga neurogênica hiperativa pós acidente vascular encefálico. Trabalho de Conclusão de Curso (Graduação em Fisioterapia) - Universidade Federal de Uberlândia, Uberlândia.

Araújo, T.G. (2017). Tratamento da síndrome da bexiga hiperativa neurogênica feminina na doença de Parkinson através da estimulação transcutânea do nervo tibial posterior. Universidade Federal do Rio Grande do Sul. Faculdade de Medicina. Programa de Pós-Graduação em Medicina: Ciências Médicas.

Barroso, U., Tourinho, R., Lordêlo, P., Hoebeke, P. \& Chase, J. (2011). Electrical stimulation for lower urinary tract dysfunction in children: a systematic review of the literature. Neurourol Urodyn. 30:1429-36.

Botelho, L.L.R., Cunha, C.C.D.A., \& Macedo, M. (2011). O método da revisão integrativa nos estudos organizacionais. Gestão e sociedade, 5, $121-36$. Carr, M.C. ( 2010). Conservative nonsurgical management of spina bifida. Curr Urol Rep. 11(2):109-13.

Canbaz, K.S., Kabay, S. \& Mestan et al. (2017). Long term sustained therapeutic effects of percutaneous posterior tibial nerve stimulation treatment of neurogenic overactive bladder in multiple sclerosis patients: 12-months results. Neurourol Urodyn. 36:104-110.

Chen, G., Liao, L. \& Li, Y. (2015). The possible role of percutaneous tibial nerve stimulation using adhesive skin surface electrodes in patients with neurogenic detrusor overactivity secondary to spinal cord injury. Int Urol Nephrol. 47: 451-455.

Coolen, R.L., Groen, J.M. (2019). Estimulação elétrica no tratamento das disfunções vesicais: atualização tecnológica. Evidência e pesquisa, 12 , $337-345$.

Dias, T.M. (2016). Bexiga neurogênica em crianças e adolescentes: impacto familiar e biomarcadores. Dissertação apresentada à Faculdade de Medicina da Universidade de São Paulo para obtenção do título de Mestre em Ciências Programa de Urologia Orientador. 
Dombek, K. (2019). Efeito imediato da Estimulação Elétrica Nervosa Transcutânea (TENS) nos parâmetros urodinâmicos de crianças e adolescentes com mielomeningocele. 108 f. Tese (Doutorado em Ciências)-Instituto Nacional de Saúde da Mulher da Criança e do Adolescente Fernandes Figueira, Fundação Oswaldo Cruz, Rio de Janeiro.

Guys, J.M., Haddad, M., Planche, D., Torre, M., Louis C. \& Breaud J. (2004). Sacral neuromodulation for neurogenic bladder dysfunction in children. J Urol. 172(4 Pt 2):1673-6.

Hearn, J.H., Selvarajah, S., Kennedy, P. \& Taylor, J. (2018). Stigma and self-management:an Interpretative Phenomenological Analysis of the impact of chronic recur-rent urinary tract infections after spinal cord injury. Spinal Cord Ser Cases. 4:12.

Jahromi, M.S., Mure, A. \& Gomez, C.S. (2014). UTIs in Patients with Neurogenic Blad-der. Curr Urol Rep;15.

Kabay, S. et al. (2016). The Clinical and Urodynamic Results of Percutaneous Posterior Tibial Nerve Stimulation on Neurogenic Detrusor Overactivity in Patients with Parkinson's Disease. Urology, v. 87, p. 76-81.

Knüpfer, S.C., Liechti, M.D. \& Mordasini L., et al. (2014). Protocol for a randomized, placebo-controlled, double-blind clinical trial investigating sacral neuromodulation for neurogenic lower urinary tract dysfunction. BMC Urol. 4:65

Leal, F.L. (2018). Avaliando a bexiga neurogênica infantil com a CIF. 20f. Trabalho de Conclusão de Curso (Graduação em Fisioterapia) - Departamento de Fisioterapia, Universidade Federal do Rio Grande do Norte, Natal..

Leng, W.W. \& Chancellor, M.B. (2005). How sacral nerve stimulation neuromodulation works. Urol Clin North Am. 32:11-8.

Liao, L. (2015). Evaluation and management of neurogenic bladder: What is new in China? International Journal of Molecular Sciences, v. 16, n. 8, p. $18580-18600$.

Lue, T.F. \& Tanagho, E.A. (2014). Distúrbios neurológicos da bexiga. In. SMITH.; TANAGHO, E. A. Urologia geral: ed. 18. Porto Alegre: AMGH, p. 441456.

Maternik, M., Krzeminska, K. \& Zurowska, A. (2015). The management of childhood urinary incontinence. Pediatr Nephrol. 30:41-50.

Mendes, K.D.S., Silveira, R.C.D.C.P., \& Galvão, C.M. (2008). Revisão Integrativa: método de pesquisa para a incorporação de evidências na saúde e na enfermagem. Texto and contexto enfermagem, 17, 758-64

Monteiro, L.M., Cruz, G.O., Fontes, J.M., Vieira, E.T, Santos, E.N \& Araújo G.F, et al. (2017). Early treatment improves urodynamic prognosis in neurogenic voiding dysfunction: 20 years of experience. J Pediatr (Rio J). 93:420---7.

Mcdonough, S. \& Kitchen, S. (2003). Estimulação elétrica neuromuscular e muscular. In: Eletroterapia: Prática Baseada em Evidências. 2o ed Manole; 2003. p. 350.

Nepomuceno, E.A. (2013). Análise dos resultados da eletroestimulação neural transcutânea parassacral e oxibutinina no tratamento de crianças com bexiga neurogênica.

Nijman, R. (2016). Diagnosis and management of urinary incontinence in childhood. In: The 6th International Consultation on Incontinence. 6th ed Tokyo: ICIUD; p. 2636.

Ojha, R., George, J., Chandy, B. R., Tharion, G. \& Devasahayam, S. R. (2015). Neuromodulation by surface electrical stimulation of peripheral nerves for reduction of detrusor overactivity in patients with spinal cord injury: A pilot study. The Journal of Spinal Cord Medicine. 38(2):207-213.

Perissinotto, M.C, Ancona, C.L., Lucio, A., Campos, R.M. \& Abreu, A. (2015). Transcutaneous tibial nerve stimulation in the treatment of lower urinary tract symptoms and its impact on health-related quality of life in patients with parkinson disease. J Wound Ostomy Continence Nurs. 42:94-9.

Radziszewski, K. (2013). Outcomes of electrical stimulation of the neurogenic bladder: Results of a two-year follow-up study. NeuroRehabilitation, v. 32, n. 4, p. $867-873$.

Rocha, J.N. (2009). Fundamentos da Eletroterapia Aplicados ao Trato Urinária. In: Aplicações clínicas das técnicas fisioterapêuticas nas disfunções miccionais e do assoalho pélvico. $1^{\circ}$ ed Campinas: Personal Link Comunicações.

Schops, T.F, Schneider, M.P., Steffen, F. \& Ineichen, B.V, et al. (2015). Neurogenic lower urinary tract dysfunction (NLUTD) in patients with spinal cord injury: longterm urodynamic findings. BJU International. 115(Suppl 6):33-38.

Schneider, M. P. et al. (2015). Tibial nerve stimulation for treating neurogenic lower urinary tract dysfunction: A systematic review. European Urology, v. 68 , n. 5 , p. $859-867$.

Stampas, A., Korupolu, R., Zhu, L., Smith, C.P. \& Gustafson, K. (2019). Safety, feasibility, and efficacy of transcutaneous tibial nerve stimulation in acute spinal cord injury neurogenic bladder: a randomized control pilot trial. Neuromodulation. 22(6):716-22.

Starkman, J., Smith, C.P. \& Staskin, D.R. (2010). Surgical options for drug-refractory overactive bladder patients. Rev Urol. 12:97-110.

Stepherson, R.G. \& Shelly, E.R. (2010). Electrical Stimulation and Biofeedback for Genitourinary Dysfunction. In: Clinical Electrophysiology: Electrotherapy and Electrophysiologic Testing. 3o ed Porto alegre: Artmed. p. 560.

Tsang, B., Stothers, L., Macnab, A., Lazare, D. \& Nigro, M. (2016). A systematic review and comparison of questionnaires in the management of spinal cord injury, multiple sclerosis and the neurogenic bladder. Neurourol Urodyn. 35(3):354-64

Zecca, C. et al. (2016). Posterior tibial nerve stimulation in the management of lower urinary tract symptoms in patients with multiple sclerosis. International Urogynecology Journal, v. 27, n. 4, p. 521-527. 